\title{
Case Series of Antrovestibular Polyp: An Unusual Growth of Antral Polyp Toward the Nasal Vestibule Through the Anterior Fontanelle
}

\author{
Goun Choe, $\mathrm{MD}^{1}$, January E. Gelera, $\mathrm{RPh}, \mathrm{MD}^{2}$, and Jeong-Whun Kim, $\mathrm{MD}, \mathrm{PhD}^{1}$ \\ ${ }^{1}$ Department of Otorhinolaryngology-Head and Neck Surgery, Seoul National University College of Medicine, Seoul National University \\ Bundang Hospital, Seongnam, Korea; and ${ }^{2}$ Department of Otorhinolaryngology-Head and Neck Surgery, \\ University of Santo Tomas Hospital, Manila, Philippines
}

Background and Objectives: This case series is aimed to introduce a new term, antrovestibular polyp (AVP), for an antral polyp herniating anteriorly toward the nasal vestibule and to describe an antral polyp direction of growth through the anterior and posterior fontanelles.

Materials and Method: This is a retrospective study involving review of patients who underwent surgery due to maxillary sinus polyp herniating anteriorly toward the nasal vestibular area or posteriorly toward the choana at a tertiary training hospital from January 2007 through July 2016. Their demographic data, computed tomography scan findings, and endoscopic evaluations were analyzed.

Results: This study included 49 subjects; 8 (16.33\%, 6 males) with AVP and 41 (83.67\%, 24 males) with antrochoanal polyps (ACP). The mean ages of AVP and ACP patients were 9 and 14.4 years, respectively $(\mathrm{p}=0.006)$. The subjects were identified as AVP when computed tomography scan showed an antral polyp directed anteriorly toward the nasal vestibular area, while polyps growing toward the choana were identified as ACP. Endoscopic review showed that AVP grew out through an accessory ostium located anterior to the uncinate process at the area of the anterior fontanelle, while ACP started from an accessory ostium of the posterior fontanelle or a widened maxillary natural ostium.

KEY WORDS: Antrovestibular polyp · Antrochoanal polyp · Antral cyst · Accessory ostium · Maxillary sinusy.

\section{INTRODUCTION}

Dutch anatomist, Ruysch first described nasal polyps arising from antrum in 1691. ${ }^{12)}$ Similarly, Zuckerkandl, a Hungarian-Austrian anatomist in 1891, reported a case of polyp from the maxillary sinus with herniation through a widened accessory ostium. ${ }^{13)}$ In 1906, Killian published a paper "The origin of choanal polypi" describing a polyp originating in the maxillary sinus, which is usually unilateral and solitary that extends into the posterior choana of the nasal cavity. ${ }^{144)}$
Through the years numerous papers have been published about Killian's polyp or antrochoanal polyp (ACP). However, there has been no written report of an unusual variant of the maxillary sinus polyp that herniates through a dehiscence or accessory ostium of the anterior fontanelle towards the nasal vestibular area, and not towards the choana. For this reason, the study is aimed to introduce a new terminology for this unrecognized presentation of polyp as "antrovestibular polyp (AVP)" and to describe the direction of AVP or ACP growth through the anterior or posterior fon-

Received: April 19, 2019 / Revised: June 12, 2019 / Accepted: June 20, 2019

Address for correspondence: Jeong-Whun Kim, MD, PhD, Department of Otorhinolaryngology-Head and Neck Surgery, Seoul National University College of Medicine, Seoul National University Bundang Hospital, 173-82 Gumi-ro, Bundang-gu, Seongnam 13620, Korea

Tel: +82-31-787-7405, Fax: +82-31-787-4057, E-mail: kimemails7@gmail.com

Address for correspondence: January E. Gelera, RPh, MD, Department of Otorhinolaryngology-Head and Neck Surgery, University of Santo Tomas Hospital, España Street, Sampaloc, Manila 1015, Philippines

Tel: +63-731-3001; 2224, E-mail: vignettejan@gmail.com 
tanelle.

\section{METHODS}

\section{Subjects}

This is a retrospective study involving a review of patients who underwent surgery due to maxillary sinus polyp herniating anteriorly toward the nasal vestibular area or posteriorly toward the choana at a tertiary training hospital from January 2007 through July 2016. This study described the unique clinical presentation of AVP and then compared it to the more common antral polyp, ACP.

\section{Computed tomographic and nasal endoscopic evaluation}

All patients had computed tomography (CT) scans of the paranasal sinuses. Diagnosis of AVP was made when a polypoid opacity originating from the maxillary sinus extended through a defect of the anterior fontanelle which is located inferior and anterior to the uncinate process, ${ }^{5)}$ toward the nasal vestibular area (Fig. 1). On the other hand, diagnosis of ACP was given to a polypoid opacity of the maxillary sinus protruding through a posterior fontanelle defect, whose boundaries include the posterior end of the uncinate process anteriorly and the palatine bone posteriorly, ${ }^{5)}$ then extending posteriorly toward the choana (Fig. 2). The au- thors of this study reviewed the CT scans and compared the position of the antral polyp with the uncinate process in axial, coronal, and sagittal view using the 3D navigation function. Through this, it was judged whether the polyp was from accessory ostium or natural ostium.

At the same time, nasal endoscopic examination was also used for diagnosis. For AVP, intra-operative pictures revealed a defect or presence of an accessory ostium in the anterior fontanelle where polyps came out of the maxillary sinus (Fig. 1) while for ACP a polyp was seen herniating from an accessory ostium of the posterior fontanelle or from a widened natural ostium (Fig. 2).

Additionally, the presence of paranasal anatomic variations and chronic rhinosinusitis were identified. ${ }^{6}$ Preoperative, intraoperative and postoperative sinonasal endoscopic photographs were taken for all subjects. The polyp location in relation to the fontanelles and the presence of an accessory ostium or defect was recorded.

\section{Operational details and pathologic analysis}

All the patients underwent routine endoscopic sinus surgery for removal of the polyps. The origin of the polyp was observed via 30 degree and 70 degree endoscope during the surgery for complete removal.

For pathologic analysis, pathological results of polyps were analyzed by their medical records.

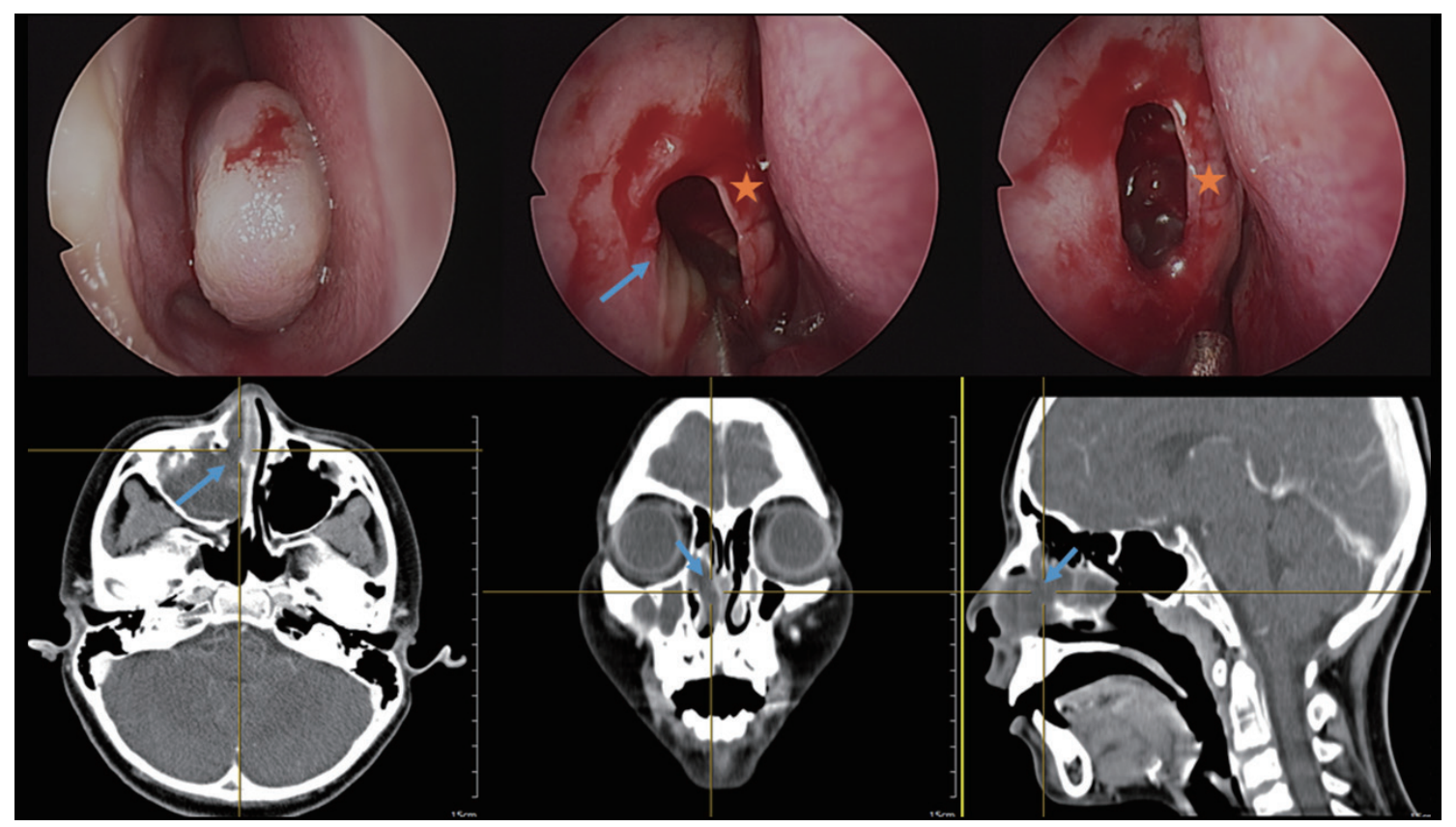

Fig. 1. Antrovestibular polyp. The crosshairs on the radiographs points to the antrovestibular polyp in different cross-sectional planes. In the endoscopic photographs, the arrow indicates the defect in the anterior fontanelle and the star marks indicate the uncinate process shown after antrochoanal polyp was removed. 


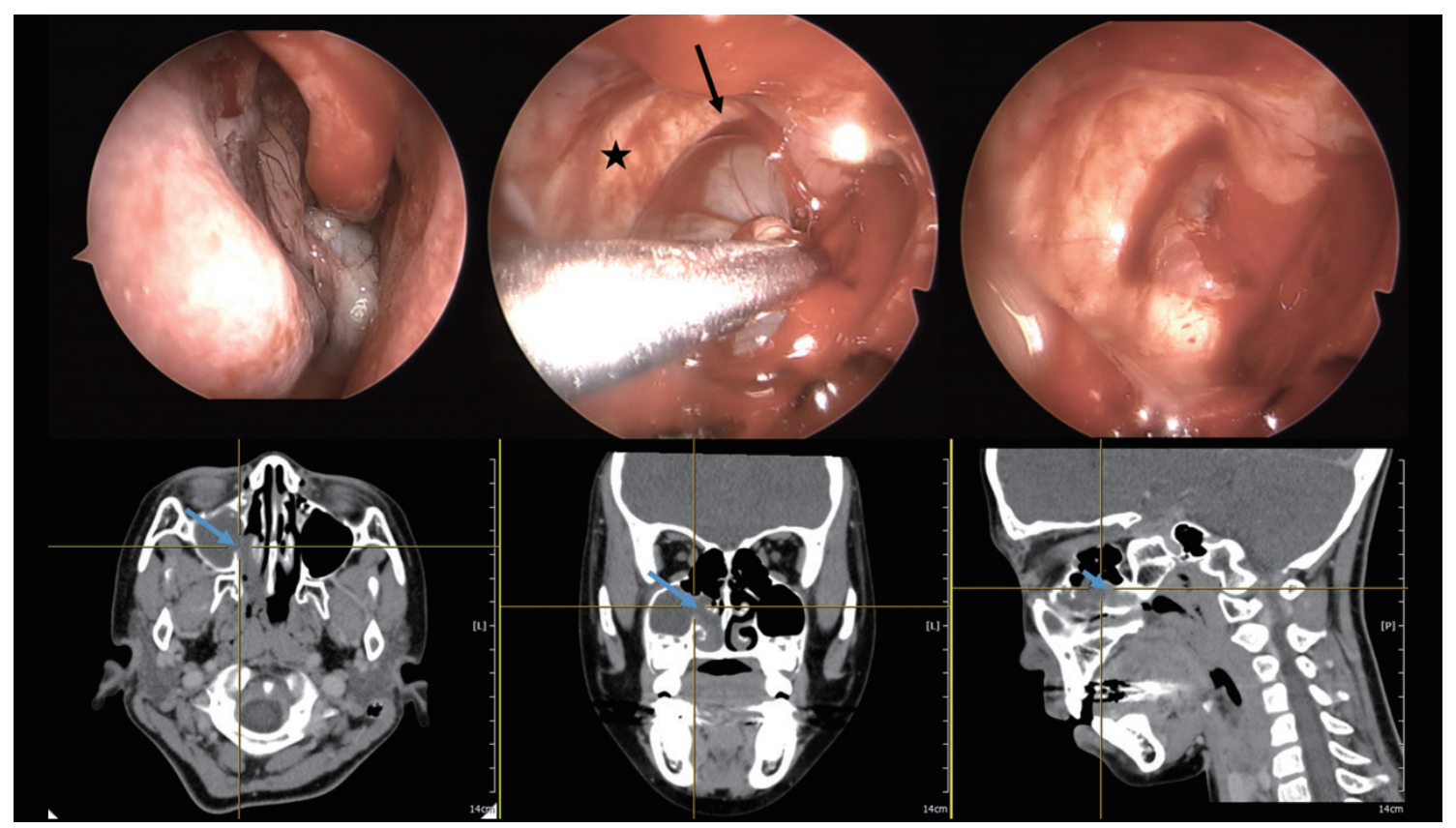

Fig. 2. Antrochoanal polyp. In the computed tomography scans, the crosshairs and arrows indicate the antrochoanal polyp. In the endoscopic photographs, the star mark indicates the large defect in the posterior fontanelle and the arrow indicates the antrochoanal polyp.

Table 1. Summary of comparison between antrochoanal polyp and antrovestibular polyp

\begin{tabular}{lcc} 
& Antrochoanal polyp & Antrovestibular polyp \\
\hline Number of subjects & 41 & 8 \\
M:F ratio & $24: 17$ & $6: 2$ \\
Average age & 14.4 & 9 \\
Passing ostium & Posterior fontanelle, natural ostium & Anterior fontanelle \\
Direction of polyp & Choana, nasopharynx (Posterior) & Nasal vestibule, nasal sill (Anterior) \\
\hline
\end{tabular}

\section{RESULTS}

This study included 49 subjects; 8 patients $(16.3 \%, 6$ males) had AVP and 41 (83.6\%, 24 males) had ACP. The mean age of AVP and ACP was 9 (range: 8-12) and 14.4 years (range: $4-46)$, respectively ( $\mathrm{p}=0.006)$ (Table 1$)$.

For AVP, a glistening white polyp was noted abutting the nasal sill while for ACP; the polyp was noted in the middle meatus extending into the nasopharynx.

Among the 8 AVP subjects, 3 (40\%) presented with concomitant bilateral chronic rhinosinusitis. Five children had ipsilateral septal deviation, 3 had Haller cells. There was no concha bullosa observed.

In pathologic findings, seven subjects of AVP and 33 of ACP had the pathological results after the operation. One of AVP and eight of ACP did not undergo pathological examination. All of the seven AVP were lymphoplasma-dominant polyps. In ACP, 31 was lymphoplasma-dominant and two were eosinophilic polyps.

\section{DISCUSSION}

According to Berg and colleagues, ACP could arise from an antral cyst, which is present in $8-10 \%$ of the population. ${ }^{7)}$ Chen's study on the other hand suggested that development of ACP occurs from obstruction of acinar mucous gland as a consequence of chronic inflammatory condition like allergic rhinitis or infectious rhinitis. ${ }^{8)}$ The others proposed intramural cyst from tooth migration and lymphatic duct stenosis following rhinosinusitis as the origin of antral cyst. ${ }^{19)}$ The authors in this study believe that AVP follows the same pathogenesis as ACP.

We also consider that AVP herniation into the nasal cavity is influenced by the same forces as proposed by Frosini in his study about ACP. In the study, chronic inflammation (allergic or infectious) initiated antral cyst formation and 
mucosal edema leading to ostiomeatal complex or middle meatus obstruction. As air is trapped inside the maxillary sinus, high pressure develops forcing the retention cyst out into the nasal cavity. ${ }^{1)}$

The difference between AVP and ACP lies in the direction of antral polyp herniation into the nasal cavity. It is assumed that the anterior or posterior protrusion of antral polyps is mainly influenced by the anatomic location of the accessory ostium in relation to the fontanelles. The accessory ostium of the maxillary sinus may be formed either congenitally or acquired because of chronic maxillary sinusitis or middle meatal pathology. ${ }^{1011)}$ All of our patients presented with ostiomeatal complex obstruction.

In pathologic analysis, all the AVP that had pathologic examination were lymphoplasma-dominant polyps. In $33 \mathrm{sub}-$ jects of pathologically diagnosed ACP patients, 31 (93.94\%) were lymphoplasma-dominant and 2 (6.06\%) were eosinophilic. They have similar pathologic findings in general, and more accurate data will be obtained if a large amount of patient data is accumulated. Therefore, it is thought that AVP and ACP have pathologically similar origin but only different growing direction.

The prevalence of accessory ostium varies from different studies conducted either on cadavers or live subjects. In these studies, the reported prevalence ranges from $0-$ $43 \%{ }^{10) 12)}$ The prevalence would be higher in patients with nasal pathology like rhinosinusitis. ${ }^{13) 14)}$ Another study identified accessory ostium in $4.5 \%$ of healthy population and $25 \%$ among subjects with chronic rhinosinusitis. ${ }^{5)}$ Though most of the studies in the list did not specify the location of accessory ostium in relation to the fontanelles, some authors reported their presence either in anterior or posterior nasal fontanelle. It is a widely accepted knowledge that the accessory ostium is more common in posterior fontanelle. ${ }^{5) 13) 15 \text { ) }}$ However, there were studies that reported a higher occurrence of anterior fontanelle defect than in the posterior fontanelle. ${ }^{10) 12) 16-18)}$

In conclusion, the present study demonstrates that AVP is another variant of antral polyp herniating through a congenital or acquired defect of anterior fontanelle. Anatomically, the accessory ostium of the posterior fontanelle is lo- cated inferiorly and posteriorly from the natural ostium, which could explain the posterior course of ACP in the nasal cavity. In contrast, AVP leaves the maxillary sinus via a defect in the anterior fontanelle, which the authors of the current study believe as the reason for the trajectory of polyp towards the nasal vestibule.

\section{REFERENCES}

1) Frosini P, Picarella G, de Camporora E. Antrochoanal polyp analysis of 200 cases. ACTA Otorhinolaryngologica Italica 2009;29:21-6.

2) Ruysch F. Observation um anatomica chirurgicaram anturca;1691.

3) Zuckerkandl E. Normale und pathologische Anatomie der Nasenholme. Vienna; 1892.

4) Killian G. The origin of choanal polypi;1906. p.81-2.

5) Stammberger HR, Kennedy DW, Anatomic Terminology G. Paranasal sinuses:anatomic terminology and nomenclature. Ann Otol Rhinol Laryngol Suppl 1995;167:7-16.

6) Ashraf N, Bhattacharyya N. Determination of the incidental LundMackay score for the staging of chronic rhinosinusitis. Otolaryngol Head Neck Surg 2001;125:483-6.

7) Berg O, Carenfelt C, Silfversward C, Sobin A. Origin of the choanal polyp. Arch Otolaryngol Head Neck Surg 1988;114(11):1270-1.

8) Chen JM, Schloss MD, Azouz ME. Antro-choanal polyp: a 10-year retrospective study in the pediatric population with a review of the literature. J Otolaryngol 1989;18(4):168-72.

9) Berg O, Carenfelt C, Sobin A. On the diagnosis and pathogenesis of intramural maxillary cysts. Acta Otolaryngol 1989;108(5-6):464-8.

10) Singhal M, Singhal D. Anatomy of accessory maxillary sinus ostium with clinical application. International Journal of Medical Science and Public Health 2014;3(3):327.

11) Levine H, Mark M, Rontal M, Rontal E. Complex anatomy of lateral nasal wall simplified for endoscopic sinus surgery. New York: Thieme Medical Publishers;1993.

12) Kumar H, Choudhry R, Kakar S. Osteomeatal complex obstruction is not associated with.pdf;2011.

13) Lund V. Anatomy of the nose and paranasal sinuses. 6th ed: Oxford: Butterworth Heinemann;1997.

14) Jog M, McGarry GW. How frequent are accessory ostia? The Journal of Laryngology and Otology 2003;117:270-2.

15) Mladina R, Skitarelic N, Casale M. Two holes syndrome (THS) is present in more than half of the postnasal drip patients? Acta Oto-Laryngolica 2010;130:1274-77.

16) Mahajan A, Mahajan A, Gupta K, Verma P, Lalit M. Anatomical variations of accessory maxillary sinus ostium: an endoscopic study. Int J Anat Res 2017;5(1):3485-90.

17) Bharathi D, Komala B, Sharath R. Anatomical Study of Accessory Maxillary Ostia and Its Surgical Importance. International Journal of Healthcare Sciences 2015;2(2):176-9.

18) Patil M, Manjunath KY. Ostium maxillare accessarium-a morphologic study. National Journal of Clinical Anatomy 2012;1:171-5. 